\section{A small pulse generator for calibrating EEG recording systems*}

WILLIAM J. MUNDL

Allan Memorial Institute, McGill University, Montreal, Quebec, Canada

A battery-powered pulse generator of uncomplicated circuit arrangement has the advantage of being easily constructed and, also, can be positioned in a confined testing area in order to simulate the low-amplitude signals of a $\mathrm{S}$. It may be especially useful for EEG recording systems for small animais. In this way, the complete recording system, including connecting cables, can be calibrated, and its performance readily assessed with respect to frequency response and to susceptibility to noise pickup.

In situations where EEG is recorded from moving $\mathrm{Ss}$, the electrical connections are often complicated by long cables and commutators. This situation makes it desirable to have the calibration source connected directly to cable ends at the S's location to facilitate an evaluation of the complete recording system. The instrument described here is batterypowered and of small dimension and may therefore be placed into any test enclosure for simulating the experimental gathering of EEG signals. A similar calibration instrument which generates simulated neuronal pulses has been previously outlined (Mundl, 1970).

The nonlinear waveforms generated by the calibrator (square wave, rectangular pulse, and impulse) are useful for visualizing the linear distortions which a physiological signal undergoes when it passes through an amplification system of limited frequency response. The frequency response is purposely limited in practically every EEG recording system, in order to improve the signal-to-noise ratio. The latter is a function of the frequency bandwidth and is proportional to the square root of the pass-band. High. or low-frequency cutoffs may be gradual, as achieved with a single RC combination, or very sharp, as in the case of a bandpass filter.

\section{USE OF THE \\ PULSE GENERATOR}

The pulse generator is intended for use as a quick check on the amplifier's gain and for visualizing distortions impressed upon a nonlinear waveform by frequency-dependent circuits. It must be borne in mind that such circuits do not distort a sine wave but can only change its amplitude and phase relation between input and

*Development of this apparatus was supported by grants to Dr. R. B. Malmo from the National Research Council and Defence Research Board of Canada and U.S. National Institute of Mental Health. output. Three types of waveforms are available from the pulse generator, namely a square wave, a rectangular pulse, and an impulse. Such waveforms undergo a high level of distortion due to low-frequency limiting in the amplifier. Figure 1 shows the output waveforms of an amplifier in their deviation from the applied signals. The different time constants (RC) determine the amount of frequency limiting at the low and the high end of the pass-band of the amplifier. Time constant and frequency attenuation are related as $R C=1 / 2 \pi f$, f denoting the frequency (sinusoidal) at $3 \cdot d B$ attenuation of peak amplitude. Designating the time constants in Fig. 1 as short, medium, and long is arbitrary and depends on the level of amplitude attenuation or on the amount of distortion to nonlinear waveforms tolerated by the $\mathrm{E}$. As pointed out above, the procedure is always one of compromise based on the signal-to-noise ratio.

The amplitude decay of the high-pass follows an exponential form. The instantaneous output voltage is $v=V \exp (-t / R C)$, where $V$ is the peak voltage and RC the time constant. The output voltage, $v$, falls to $37 \%$ of $\mathrm{V}$ at a time $t=R C$. In the low-pass, the exponential rise of the output voltage is: $v=V[1-\exp (-t / R C)]$. Similarly, as in the low-pass, the output voltage reaches $63 \%$ of the applied voltage at time $t=R C$

The impulse function, defined theoretically as a pulse having an infinite amplitude and an infinitesimal duration, is approximated by generating it through differentiation of a step function, in this case a rectangular pulse. The impulse is useful for assessing both high-frequency and low-frequency response and especially for indicating the recovery time of the amplifier from overvoltages, which are frequently encountered during recording in the form of transients.

The tolerable extent of the distortion effect of the low-pass and the high-pass filtering on the EEG
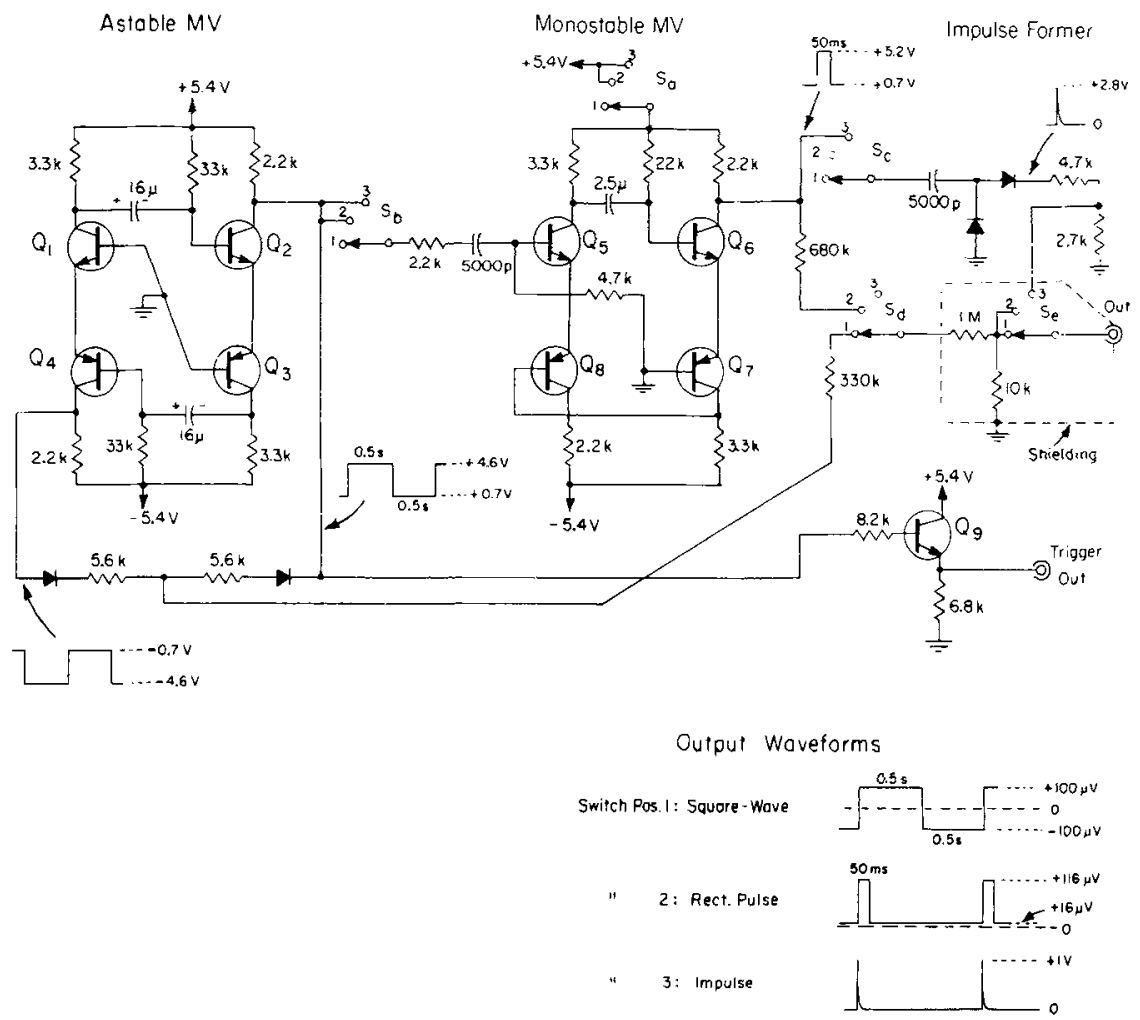

Fig. 1. Changes in shape of a nonlinear waveform due to frequency limiting in a recording system. Sinusoidal waveforms are not distorted by frequency limiting, but are merely changed in their amplitude or their phase relation. 


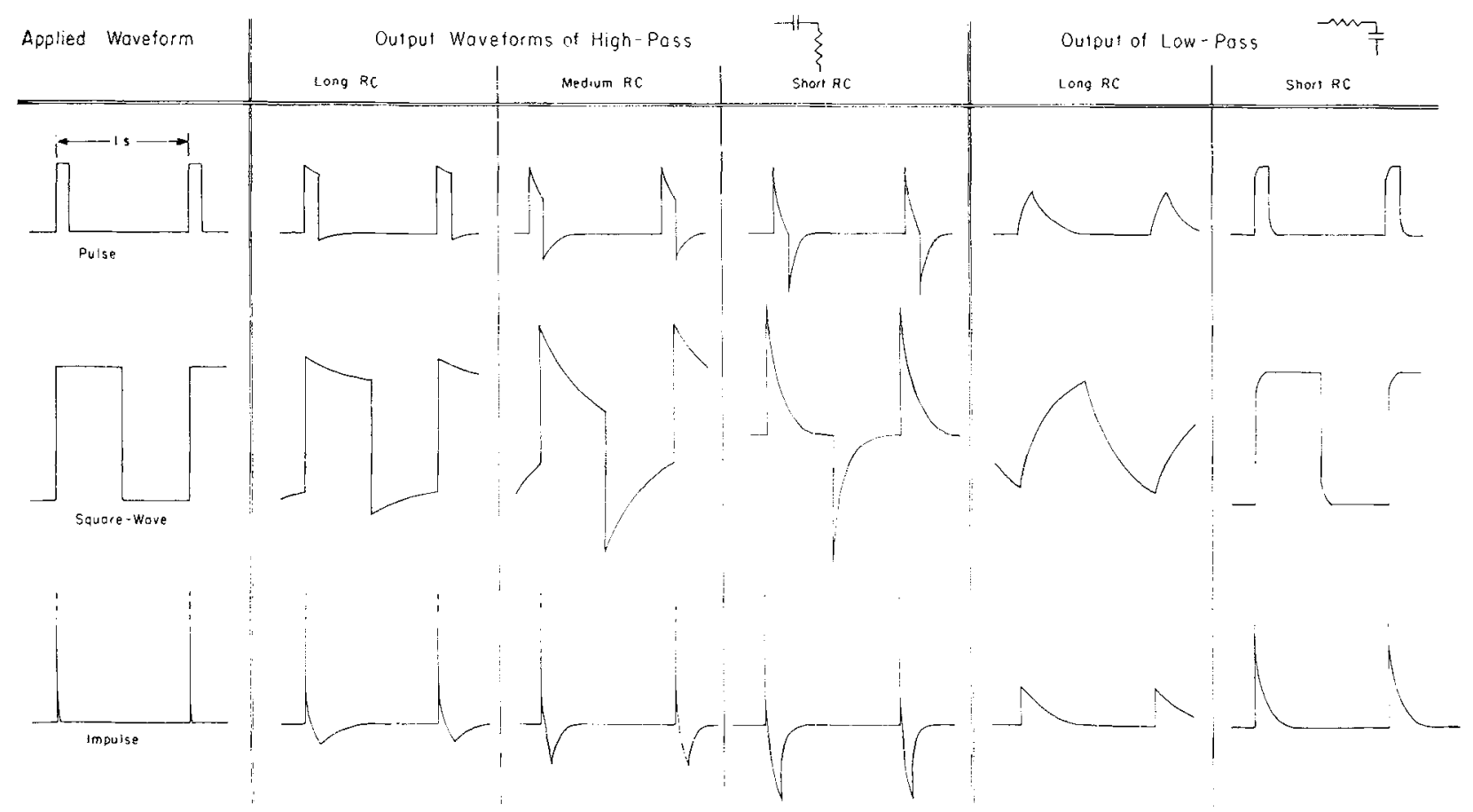

Fig. 2. Circuit diagram. The astable multivibrator (MV) generates the square wave and also triggers the monostable MV, which supplies the rectangular pulse. The trigger output provides for synchronized averaging of a wa veform in an averaging computer.

signal depends on the purpose of the experiment and remains a prerogative of the E's judgment. A considerable volume of recorded EEG signals appear sinusoidal and may perhaps be considered as such in origin. In this case, frequency limiting will not cause a distortion, but will only change its amplitude or phase relation. Conversely, an EEG waveform can also result from a sharply defined burst of unit activity. Then the envelope of this burst will resemble a rectangular pulse, and the output waveform of the amplifier will consist of a positive-negative swing. This would apply to signal patterns obtained in the manner of "evoked responses."

\section{CIRCUIT $^{1}$}

Sharply defined square waves and rectangular pulses are achieved by using the circuit configuration of the multivibrators (MV) shown in Fig. 2. The astable MV consists of two grounded-base stages, $Q_{1}$ and $Q_{3}$, which provide the loop gain necessary for remaining in the switched states. $Q_{2}$ and $Q_{4}$ are emitter followers which furnish impedance matching between the high output impedance of the collector of the grounded-base stage and the low input impedance of the emitter of the complementary grounded-base stage. Including collector resistors in the emitter followers limits the collector current in the saturated state and thereby supplies a cleanly switched output waveform. The $33 \cdot \mathrm{k} \cdot \mathrm{ohm}$ resistors and 16-microF capacitors serve as timing elements.

In the monostable MV, which works in a similar manner to the astable $\mathrm{MV}$, the pulse length is determined by the $22-\mathrm{k}-\mathrm{ohm}$ resistor and the 2.5-microF capacitor, which in turn provide the exponential decay waveform to retain $Q_{6}$ and $Q_{7}$ in the cutoff state for a given time. A description of such multivibrators was given by Pugh (1965).

Positive and negative outputs of the astable MV are combined over two diodes to furnish a square wave with equal positive and negative voltage swing, which, after attenuation to 200 microV peak-to-peak, is available at the output jack. The rectangular pulse of the monostable $\mathrm{MV}$ is attenuated to 100 microV and is switched on to the output at Switch Position 2. The impulse is formed by differentiation of the rectangular pulse in the impulse former of Fig. 2. In order to reduce battery drain, Switch Section $S_{a}$ switches off the positive supply voltage from the monostable MV when only the square-wave output is needed. The attenuation network switch section, $S_{e}$, should be carefully shielded to eliminate crosstalk from adjacent switch sections.

In a case where the EEG recording system includes an averaging computer, the averaging process can be simultaneously observed and calibrated by using the trigger output.

\section{REFERENCES}

MUNDL, W, J. Simulated unit pulses for calibrating. Behavior Research Methods \& Instrumentation, 1970, 2, 139 .

PUGH, A. Zero phase-shift multivibrators. Electronic Engineering, 1965, 37, 24-25.

\section{NOTE}

1. List of major parts: Mercury batteries (2)-Mallory, Type 302904; NPN transistors (5)-Texas Instruments. Ty pe 2N 3704 ; PNP transistors (4)-Texas Instruments, Type 2N 3702; diodes (4)-Philips, Type OA 200; switch (1)-Centralab, Type PA 1018. 